\title{
Identity Based Self Delegated Signature - Self Proxy Signatures
}

\author{
S. Sharmila Deva Selvi, S. Sree Vivek, S.Gopinath, C. Pandu Rangan \\ TCS Lab, Department of CSE, \\ Indian Institute of Technology Madras (IITM) \\ Chennai, India \\ Email: \{sharmila,svivek,gopinath,prangan\}@cse.iitm.ac.in
}

\begin{abstract}
A proxy signature scheme is a variant of digital signature scheme in which a signer delegates his signing rights to another person called proxy signer, so that the proxy signer can generate the signature of the actual signer in his absence. Self Proxy Signature (SPS) is a type of proxy signature wherein, the original signer delegates the signing rights to himself (Self Delegation), there by generating temporary public and private key pairs for himself. Thus, in SPS the user can prevent the exposure of his private key from repeated use. In this paper, we propose the first identity based self proxy signature scheme. We give a generic scheme and a concrete instantiation in the identity based setting. We have defined the appropriate security model for the same and proved both the generic and identity based schemes in the defined security model.
\end{abstract}

Keywords: Identity Based Cryptography, Self-Proxy Signatures, Delegation, Random Oracle Model.

\section{INTRODUCTION}

The notion of proxy signature schemes dates back to 1996, proposed by Mambo, Usuda and Okamoto in their seminal paper [14]. In proxy signature scheme, a user Alice, called the original signer delegates her signing rights to another user Bob, called the proxy signer. A verifier can distinguish between a normal signature and a proxy signature but then is convinced that the message is authenticated by Alice. Proxy signatures have a number of applications, including e-commerce, mobile agents and distributed shared objects. The original signer Alice sends a signature on the message warrant which consists the rules governing the delegation to Bob the proxy signer. Bob can now generate a new proxy private key with the help of Alice and sign on behalf of Alice. In 1998, Oded Goldreich, Birgit Pfitzmann and Ronald L. Rivest [5] introduced delegation schemes where a user delegates certain rights to himself. Their motivation was, even though a user has a long-term permanent key, which is used to receive some personalized access rights, the user may wish to delegate these rights to a new temporary possibly short-term keys which he creates to use on his laptop when on travel, to avoid having to store his primary secret key on the vulnerable laptop. They have succeeded without relying on special-purpose (e.g., tamper-proof) hardware installed in the laptop and have proposed several schemes. However, their schemes work for signatures in the Public Key Infrastructure (PKI) setting. In Self Proxy Signature (SPS), a user delegates his signing rights to himself, i.e. the user can generate multiple pairs of temporary public and private keys. The lifetime of the temporary keys can be controlled by creating proper message warrants, depending on the application.

\section{A. Motivation}

Self proxy signatures are used in scenarios where the user wants to create new key pairs from the existing key pair. The newly generated key pair is called as "Temporary Key" pair and the existing key pair from which the Temporary key pair is generated is called as "Permanent Key" pair. It is important to note that while permanent key pair is generated by PKG and it is done only once per user, the temporary key pair is generated by the user and can be done any number of times. We explain three situations, where self delegation is useful and these situations commonly arise in practice.

To reduce the probability of exposure of the permanent private key: Nowadays, numerous internet services such as, internet banking, home trading, on-line payments, electronic commercial services and other secure online transactions rely on Public Key Cryptography. Public key cryptography plays an important role in the authentication of users in these systems. This causes a potential security threat, namely "increase in the probability of the permanent private key being exposed". For instance, if the permanent private key is used in an insecure computing environment such as a public PC or a friend's PDA, a malicious program can plunder the private key by searching the memory where the private key is stored, or by hijacking the password for decrypting the enciphered private key. Moreover, this gives room to access any other online services of the user, which rely on this plundered permanent private key. It is to be noted that this situation was discussed in [12].

To create weak Temporary Keys means less number of bits: This situation is common in secure communication protocols such as SSL/TLS, where the session key exchange between a server and its client is done using Public Key Cryptography. When SSL was designed, United States export regulations limited RSA encryption key lengths to 512 bits for exportable applications. Unfortunately, a 512-bit permanent RSA key presents an attractive target for attack. Thus a server who wish to communicate with 
both domestic and exportable clients would like to have two keys one with 1024 bits and another key with 512 bits. This feature is called ephemeral RSA and this allows communication between an exportable client and domestic server with permanent strong key. In this scenario, the server generates a temporary 512 bit key which is signed with its strong permanent key.

To significantly improve amortized signature generation and verification cost: Besides the two reasons mentioned above, we show a significant reduction in the total signing cost during the period of validity of temporary keys. For instance, for signing $n$ messages in our scheme we may use $(2+n)$ or $(3+n)$ scalar point multiplications. While direct deployment would incur a cost of $2 n$ or $3 n$ point multiplications. More detailed comparisons are done towards the end of this paper.

Related Work: In 1996, Mambo et al introduced the concept of a proxy signature scheme [14]. Since then, many proxy signature schemes have been proposed [9][11]. The first multi-proxy signature scheme was proposed in 2000 [7]. In a multi-proxy signature scheme, an original signer could authorize a proxy signing group as his proxy agent. The proxy signature on a message on behalf of the original signer can be generated by the group members only if all the members in the proxy signing group cooperate. A contrary concept, proxy multi-signature was introduced by $\mathrm{Yi}$ et al. in 2000 [17]. A proxy multi-signature scheme is one in which a designated proxy signer can generate the signature on behalf of a group of original signers. Another kind of proxy signature scheme is multi-proxy multi-signature scheme, proposed by Hwang in [8] .

The concept of identity based cryptography was introduced by Adi Shamir in his seminal work [16] in the year 1984. The core idea of identity based cryptography is to use any arbitrary string that uniquely identifies a user as his public key. Identity based cryptography serves as an efficient alternative to Public Key Infrastructure (PKI) based systems, where the certificate management and verification of the validity of a user public key are too cumbersome. Although, the concept of self proxy was touched upon by Boldyreva et al. [2], they are not using any temporary keys for carrying out delegations. Only the permanent keys were used for both original and proxy signing and verification. This is a PKI based system. However, this system is shown to have weaknesses by Malkin et al. [13] and they proposed a new scheme based on key insulated signature schemes. To the best of our knowledge, there is no identity based self delegated signature scheme available in the literature and ours is the first attempt in this direction. Kim et al. [10] have proposed a PKI based self proxy signature scheme.

Our Contribution: Our contribution in this paper is three fold. First, we give a formal security model for identity based self proxy signatures. Next, we show that the scheme by Kim et al. [10] is existentially forgeable and finally, we propose a generic identity based self proxy signature scheme and a concrete instantiation of the same. We formally prove the security of both the generic and concrete schemes in the newly proposed security model. Both our proofs rely on the random oracle assumption.

\section{PRELIMINARIES}

We review the basic requirements and assumptions used in our paper in this section.

\section{A. Bilinear Pairing}

Let $\mathbb{G}_{1}$ be an additive cyclic group generated by $P$, with prime order $q$, and $\mathbb{G}_{2}$ be a multiplicative cyclic group of the same order $q$. A bilinear pairing is a map $\hat{e}: \mathbb{G}_{1} \times \mathbb{G}_{1} \rightarrow \mathbb{G}_{2}$ with the following properties.

- Bilinearity. For all $P, Q, R \in_{R} \mathbb{G}_{1}$ and $a, b \in \in_{R} \mathbb{Z}_{q}^{*}$

- $\hat{e}(P+Q, R)=\hat{e}(P, R) \hat{e}(Q, R)$

- $\hat{e}(P, Q+R)=\hat{e}(P, Q) \hat{e}(P, R)$

- $\hat{e}(a P, b Q)=\hat{e}(P, Q)^{a b}$

- Non-Degeneracy. There exist $P, Q \in \mathbb{G}_{1}$ such that $\hat{e}(P, Q) \neq I_{\mathbb{G}_{2}}$, where $I_{\mathbb{G}_{2}}$ is the identity element of $\mathbb{G}_{2}$.

- Computability. There exists an efficient algorithm to compute $\hat{e}(P, Q)$ for all $P, Q \in \mathbb{G}_{1}$.

\section{B. Computational Assumptions}

In this section, we review the computational assumptions related to bilinear maps that are relevant to the protocols we discuss.

1) Computation Diffie-Hellman Problem (CDHP):: Given $(P, a P, b P) \in \mathbb{G}_{1}^{3}$ for unknown $a, b \in \mathbb{Z}_{q}^{*}$, the $\mathrm{CDH}$ problem in $\mathbb{G}_{1}$ is to compute $a b P$.

Definition. The advantage of any probabilistic polynomial time algorithm $\mathcal{A}$ in solving the $\mathrm{CDH}$ problem in $\mathbb{G}_{1}$ is defined as

$$
A d v_{\mathcal{A}}^{C D H}=\operatorname{Pr}\left[\mathcal{A}(P, a P, b P)=a b P \mid a, b \in \mathbb{Z}_{q}^{*}\right]
$$

The $C D H$ Assumption is that, for any probabilistic polynomial time algorithm $\mathcal{A}$, the advantage $A d v_{\mathcal{A}}^{C D H}$ is negligibly small.

\section{Notations:}

$Q_{A}$ : An identity based public key of a user with identity $I D_{A}$.

$D_{A}$ : An identity based private key of a user with identity $I D_{A}$.

$P_{A}$ : A non-identity based public key of a user with identity $I D_{A}$ and also Temporary public of the same user.

$U_{A}$ : A non-identity based private key of a user with identity $I D_{A}$ and also Temporary private of the same user.

$P_{p u b}$ : Master public key used by the $P K G$ of the identity based system. 
$\sigma_{\text {war }}:$ An identity based warrant signature.

$\sigma_{s p}$ : A non-identity based proxy signature. $\sigma$ : Self proxy

signature which is a combination of $\left\langle\sigma_{w a r}, \sigma_{s p}\right\rangle$.

\section{REVIEW AND WEAKNESS OF SELF PROXY SignATURE SCHEME BY KIM ET AL. [10]}

We review the scheme due to Kim et al. [10] and propose the weakness of the scheme in this section. Let $\left(x_{a}, y_{a}\right)$ be the original public and private key of the signer Alice. The relation between $\left(x_{a}, y_{a}\right)$ is $y_{a}=g^{x_{a}}$.

Self Proxy Key Generation: The signer Alice chooses $k, x_{t} \in \mathbb{Z}_{q}^{*}$ and computes $r=g^{k} \bmod p$ and $y_{t}=g^{x_{t}} \bmod$ $p$. Alice computes $x_{p}=k+\left(x_{a}+x_{t}\right) H\left(m_{w}\right) \bmod q$ as the temporary self proxy private key and computes $y_{p}=g^{x_{p}}$ $\bmod p$ as the corresponding public key.

Signing: The signer Alice chooses $k^{\prime} \in \mathbb{Z}_{q}^{*}$ randomly and computes $r^{\prime}=g^{k^{\prime}} \bmod p, s^{\prime}=k^{\prime}+x_{p} H(m) \bmod q$ and sends $\left(m,\left(r^{\prime}, s^{\prime}\right), r, m_{w}, y_{t}\right)$ to the verifier Bob.

Verification: The verifier Bob recovers the self proxy public key $y_{p}$ as $y_{p}=r\left(y_{a} y_{t}\right)^{H\left(m_{w}\right)} \bmod p$ and checks whether $g^{s^{\prime}} \stackrel{?}{=} r^{\prime} y_{p}^{H(m)} \bmod p$. If the equality holds, the verifier Bob accepts $\left(r^{\prime}, s^{\prime}\right)$ as the valid self proxy signature.

\section{A. Weakness of Self Proxy Signature Scheme By Kim et al.}

The forger $\mathcal{F}$ can produce any number of forged signatures by using a single signature on a message $m$ signed by the original signer. This is shown below:

Let $s^{\prime}=k^{\prime}+x_{p} H(m) \bmod q$ be the signature on message $m$, where $r^{\prime}=g^{k^{\prime}} \bmod p$, this signature is obtained during the training phase of the forgery game.

The forger $\mathcal{F}$ divides the signature component $s^{\prime}$ with $H(m)$ and multiplies it with $H\left(m^{*}\right)$ and thus obtains $s^{*}=$ $k^{\prime} \frac{H\left(m^{*}\right)}{H(m)}+x_{p} H\left(m^{*}\right) . \mathcal{F}$ computes $r^{*}=r^{\prime \frac{H\left(m^{*}\right)}{H(m)}} \bmod p$. Now, $\left(m^{*},\left(r^{*}, s^{*}\right), r, m_{w}\right)$ is a valid signature on $m^{*}$. Hence a forgery.

\section{Generic Framework AND SECURIty Model FOR IDENTITY BASED SPS (IBSPS)}

In this section, we give the generic framework and the security model for identity based self proxy signature scheme. The basic idea behind the construction of an Identity Based Self Proxy Signature (IBSPS) scheme is to extract an identity based private key from the PKG and construct a temporary private key / public key pair using the identity based permanent private key and the system parameters. It is to be noted that the temporary key pairs are generated by user without any interaction from the PKG. The PKG works once for each user and generates permanent key of the user. The temporary public key and the warrant details for the session are signed with the identity based private key of the user and the message is signed with the corresponding temporary private key. Thus, the signature on the message consists of two components now; (a) the signature on the message with the temporary private key (b) the identity based signature on the temporary public key and the warrant details.

\section{A. Generic Framework for IBSPS}

An identity based self proxy signature scheme consists of the following nine algorithms: Setup, Extract, GenTempKey, WarrantSign, WarrantVerify, ProxySign, ProxyVerify, SPSSign and SPSVerify. The algorithms are described below:

Setup: This is a combination of an identity based system setup and a non-identity based initialize algorithm. The input to this algorithm is the security parameter $1^{\kappa}$. The PKG run's this algorithm to produce the public parameters params, which is published globally and the master private key $M s k$, kept secret by the PKG. The public parameters include a master public key $P_{p u b}$, cryptographic hash functions and the definition of the groups used in the scheme.

Extract: This algorithm is executed by the PKG. It is executed once for each user at the time of registration with the PKG. The PKG takes the master private key $M s k$ and the identity $I D_{A}$ of user $A$ as input and computes the private key $D_{A}$ corresponding to the identity $I D_{A}$.

GenTempKey: The user who wants to generate temporary private / public key pairs for various sessions executes this algorithm. The algorithm takes params as input and produces the temporary (private key, public key) pair $\left(U_{A}, P_{A}\right)$.

WarrantSign: This algorithm is executed by each user in the system to generate the signature on the message warrant $m_{w}$, which is publicly verifiable $\left(m_{w}\right.$ consists of the details regarding the duration of the delegation and the public key for the current duration). The algorithm when executed by user $A$, takes the user identity $I D_{A}$, the message warrant $m_{w}$, temporary public key $P_{A}$, the corresponding private key $D_{A}$, and params as input and outputs the identity based signature $\sigma_{\text {war }}$ on the message warrant $m_{w}$.

WarrantVerify: In order to verify the validity of the message warrant $m_{w}$, a verifier executes this algorithm. The input to this algorithm are params, the signer identity $I D_{A}$, the message warrant $m_{w}$ and the signature $\sigma_{w a r}$ on $m_{w}$ by the signer. This algorithm returns True if $\sigma_{w a r}$ is a valid signature on $m_{w}$, otherwise returns False.

ProxySign: The input to this algorithm are params, the temporary proxy private key $U_{A}$ and the actual message to be signed $m$. This algorithm is executed by the signer to generate a signature $\left(\sigma_{s p}\right)$ on $m$ using the temporary proxy private key generated by the GenTempKey algorithm.

ProxyVerify: The input to this algorithm are params, the signer identity $I D_{A}$, the temporary public key $P_{A}$ corresponding to user $A$ and the signature $\sigma_{s p}$ on message $m$. This algorithm is executed by a verifier who wants to verify 
the validity of $\sigma_{s p}$ on $m$. The output is True if $\sigma_{s p}$ is a valid signature on $m$, otherwise outputs False.

SPSSign: The self proxy signature generation algorithm is executed by the signer with identity $I D_{A}$. The input to this algorithm are the identity $I D_{A}$, the permanent private / public key pair $\left(D_{A}, Q_{A}\right)$, the temporary private / public key pair $\left(U_{A}, P_{A}\right)$, a message warrant $m_{w}$ and the message $m$ to be signed. It is to be noted that $\sigma_{w a r}$ is not executed each time during the generation of an SPS but it is executed once for each session and is reused. The signature generation procedure is given below:

- $\sigma_{w a r} \leftarrow W$ arrantSign $\left(m_{w}, P_{A}, I D_{A}, D_{A}\right)$.

- $\sigma_{s p} \leftarrow \operatorname{Proxy} \operatorname{Sign}\left(m, P_{A}, I D_{A}, U_{A}\right)$

The signature $\sigma=\left\langle m_{w}, m, \sigma_{w a r}, \sigma_{s p}\right\rangle$ is the output of this algorithm.

Note: If the warrant sign was already generated for a session by executing WarrantSign $\left(m_{w}, P_{A}, I D_{A}, D_{A}\right)$ with the temporary private / public key pair $\left(U_{A}, P_{A}\right)$, the signer can directly call ProxySign $\left(m, P_{A}, I D_{A}, U_{A}\right)$ to get $\sigma_{s p}$.

SPSVerify: In order to verify the validity of an identity based SPS $\sigma=\left\langle m_{w}, m, \sigma_{w a r}, \sigma_{s p}\right\rangle$, the verifier checks whether the message warrant $m_{w}$ is valid by checking WarrantVerify $\left(\sigma_{\text {war }}, I D_{A}, m_{w}\right) \stackrel{?}{=}$ True and whether the signature on message $m$ is valid by checking ProxyVerify $\left(\sigma_{s p}, I D_{A}, m, P_{A}\right) \stackrel{?}{=}$ True. If both the checks are valid this algorithm outputs True else it outputs False.

\section{B. Security Model for the Unforgeability of IBSPS}

Unforgeability is the most general notion of security for any digital signature scheme. Unforgeability ensures that the digital signature scheme is secure against a forger who can forge the signature of a legitimate user. The stronger notion of unforgeability is existential unforgeability against adaptively chosen messages and identity (only for identity based schemes) attacks. We propose the security model for identity based SPS in this section. The formal definition for the unforgeability of an IBSPS is defined as a game (EUF-IBSPS-CMA) between a challenger $\mathcal{C}$ and a forger $\mathcal{F}$ described below:

Setup Phase: $\mathcal{C}$ runs the Setup algorithm with the security parameter $1^{\kappa}$ and sends the system parameters params to $\mathcal{F}$.

Training Phase: $\mathcal{F}$ performs polynomially bounded number of queries, as described below in an adaptive manner (i.e., each query may depend on the responses to the previous queries).

Extract query : $\mathcal{F}$ produces an identity $I D_{A}$ as input to this oracle and obtains the identity based private key $D_{A}$ corresponding to the identity $I D_{A}$ from $\mathcal{C}$.

GetTempKey query: $\mathcal{F}$ produces an identity $I D_{A}$ and receives from $\mathcal{C}$ the temporary private, public keys $U_{A}$ and $P_{A}$ corresponding to $I D_{A}$.
WarrantSign query: $\mathcal{F}$ gives an identity $I D_{A}$, a message warrant $m_{w}$ and the temporary public key $P_{A}$ corresponding to $I D_{A}$ as input. $\mathcal{C}$ computes and returns the warrant sign $\sigma_{\text {war }}$ to $\mathcal{F}$.

ProxySign query: $\mathcal{F}$ submits a message $m$, a signer identity $I D_{A}$ and the corresponding temporary public key $P_{A}$ as input and requests the proxy sign on $m$. $\mathcal{C}$ generates the proxy signature $\sigma_{s p}$ only if $P_{A}$ was not chosen by $\mathcal{F}$ and returns $\sigma_{s p}$ to $\mathcal{F}$.

Existential Forgery: At the end of the Training Phase, $\mathcal{F}$ produces a forgery $\sigma^{*}=\left\langle m_{w}, m, \sigma_{s p}^{*}, \sigma_{w a r}^{*}\right\rangle$ for an identity $I D^{*}$ with temporary public key $P_{I D^{*}} . \mathcal{F}$ wins the EUFIBSPS-CMA game if the forgery $\sigma^{*}$ submitted by $\mathcal{F}$ meets one of the following constraints:

Case 1: - The warrant signature $\sigma_{w a r}^{*}$ is a valid forgery and $\mathcal{F}$ should not have queried the WarrantSign oracle with $\left(I D^{*}, m_{w}^{*}, P_{I D^{*}}, Q_{I D^{*}}\right)$ as input and has not queried the permanent private key corresponding to $I D^{*}$.

Case 2: - The proxy signature $\sigma_{s p}^{*}$ is a valid forgery and $\mathcal{F}$ should not have queried the ProxySign oracle with $\left(I D^{*}, m^{*}, P_{I D^{*}}\right)$ as input and has not queried the temporary private key corresponding to $P_{I D}^{*}$.

\section{Generic Identity Based SPS Scheme (Gen_IBSPS)}

In this section, we propose the generic construction for identity based SPS scheme (Gen_IBSPS) and prove the unforgeability of Gen_IBSPS. We make use of an identity based signature scheme and a non-identity based signature scheme as the basic building blocks for our generic construction. Let the identity based signature scheme be denoted as $I B S=\langle I B S$. Setup, IBS.Extract, IBS.Sig, IBS.Ver $\rangle$, and the non-identity based signature be denoted as nonIBS = 〈nonIBS.Initialize, nonIBS.KeyGen, nonIBS.Sig, nonIBS.Ver $\rangle$ As security requirements, we require both the schemes, $I B S$ and nonIBS should be existentially unforgeable under adaptive chosen message attack. Examples of $I B S$ can be one of the schemes from [4], [6], [15], [1], [16] and nonI BS can be Schnorr, EC-DSA or BLS [3] signature.

\section{A. The Generic Scheme}

As described in the previous section the generic self proxy signature scheme consists of the following nine algorithms:

$\operatorname{Setup}\left(1^{\kappa}\right)$ : The PKG publishes the system parameters params after executing IBS.Setup $\left(1^{\kappa}\right)$ and nonIBS.Initialize $\left(1^{\kappa}\right)$ algorithms.

$\operatorname{Extract}\left(I D_{A}\right): \quad$ The $\mathrm{PKG}$ executes $D_{A}=$ $I B S$. Extract $\left(I D_{A}\right)$ and sends the permanent private key $D_{A}$ to the user $A$, through a secure channel and the permanent public key $Q_{A}$ can be computed publicly. 
GenTempKey $\left(\right.$ params, $\left.I D_{A}\right)$ : This algorithm generates the temporary private / public key pair for a given identity $I D_{A}$ is obtained as $\left(U_{A}, P_{A}\right)=$ nonIBS.KeyGen $\left(I D_{A}\right)$.

WarrantSign $\left(m_{w}, P_{A}, I D_{A}, D_{A}\right)$ : The warrant signature is generated as $\sigma_{w a r}=I B S . \operatorname{Sig}\left(m_{w}, P_{A}, I D_{A}, D_{A}\right)$

WarrantVerify $\left(m_{w}, I D_{A}, \sigma_{w a r}\right)$ : Verification of the warrant signature $\left(\sigma_{\text {war }}\right)$ on the message warrant $m_{w}$ is performed as \{true,False\} $\stackrel{?}{=} I B S . V e r\left(m_{w}, P_{A}, I D_{A}, \sigma_{w a r}\right)$

$\operatorname{Proxy} \operatorname{Sign}\left(m, I D_{A}, P_{A}, U_{A}\right)$ : The proxy signature on the message $m$ by user with identity $I D_{A}$ and temporary private key $U_{A}$ is generated as $\sigma_{s p}=$ $\operatorname{nonIBS.Sig}\left(m, I D_{A}, P_{A}, U_{A}\right)$

$\operatorname{Proxy} \operatorname{Verify}\left(m, I D_{A}, P_{A}, \sigma_{s p}\right)$ : The verification of the proxy signature $\sigma_{s p}$ on message $m$ with respect to the identity $I D_{A}$ and temporary public key $P_{A}$ is performed as $\{$ True,False $\} \stackrel{?}{=}$ nonI $B S . V e r\left(m, P_{A}, I D_{A}, \sigma_{s p}\right)$

$\operatorname{SPSSign}\left(m_{w}, m, I D_{A}, P_{A}, U_{A}, Q_{A}, D_{A}\right)$ : This algorithm uses the WarrantSign and ProxySign algorithms to generate the warrant signature $\sigma_{w a r}$ and proxy signature $\sigma_{s p}$. The self proxy signature on the message $m$ and message warrant $m_{w}$ can be described as $\sigma=\left\langle m_{w}, m, \sigma_{w a r}, \sigma_{s p}\right\rangle$

$\operatorname{SPSVerify}\left(m_{w}, m, Q_{A}, P_{A}, \sigma\right)$ : The output of this algorithm is True if both True $\leftarrow$ WarrantVerify $\left(\sigma_{\text {war }}\right)$ and True $\leftarrow$ ProxyVerify $\left(\sigma_{s p}\right)$; else output False.

Note: It is to be noted that, the proof of unforgeability of Gen_IBSPS is given in the selective identity model.

\section{B. Proof of Unforgeability of Gen_IBSPS}

Theorem 1: If there exists a forger $\mathcal{F}$, who is capable of breaking the EUF-Gen_IBSPS-CMA security of the Gen_IBSPS scheme with a non-negligible advantage, then we can efficiently construct an algorithm $\mathcal{C}$, which is capable of breaking the EUF-CMA security of the underlying IBS or nonIBS scheme with the same advantage. Proof: The proof for unforgeability of the Gen_IBSPS scheme is viewed as an interactive game between algorithms $\mathcal{B}_{1}, \mathcal{B}_{2}, \mathcal{C}$ and $\mathcal{F}$, as shown in Fig. 1. Algorithm $\mathcal{B}_{1}$ represents the challenger for the $I B S$ scheme and $\mathcal{B}_{2}$ represents the challenger for the nonI $B S$ scheme. $\mathcal{B}_{1}$ and $\mathcal{B}_{2}$ challenges the algorithm $\mathcal{C}$ to forge the $I B S$ and the nonIBS systems respectively. Let $\mathcal{F}$ be a forger, who is capable of breaking the EUFGen_IBSPS-CMA security of the Gen_IBSPS scheme. Algorithm $\mathcal{C}$ can make use of $\mathcal{F}$ to forge either IBS or nonI $B S$. We briefly summarize the roles of $\mathcal{B}_{1}, \mathcal{B}_{2}, \mathcal{C}$ and $\mathcal{F}$ below.

- $\mathcal{B}_{1}$ and $\mathcal{B}_{2}$ acts as the challengers for IBS and nonI $B S$ schemes respectively.

- $\mathcal{C}$ acts as forger for both $I B S$ and nonIBS schemes.

- $\mathcal{C}$ also acts as the challenger for the forger $\mathcal{F}$. $\mathcal{F}$ is assumed to be capable of breaking the
EUF-Gen_IBSPS-CMA security of Gen_IBSPS scheme.

Setup Phase: During this phase the algorithm $\mathcal{B}_{2}$ generates a set of private / public key pairs $\left\langle P_{i}, U_{i}\right\rangle$, for $i=1$ to $n$ and sends them to the challenger $\mathcal{C}$. $\mathcal{C}$ stores the tuple $\left\langle i, P_{i}, U_{i}\right\rangle$ in a list $F_{1} \cdot \mathcal{B}_{1}$ challenges $\mathcal{C}$ to generate a forgery of an $I B S$ signature for the identity $I D^{*}$ on any arbitrary message. $\mathcal{B}_{2}$ challenges $\mathcal{C}$ to generate a forgery of a nonIBS signature for the identity $I D^{*}$ with temporary public key $P_{I D^{*}} \cdot \mathcal{C}$ gives $I D^{*}$ and $P_{I D^{*}}$ to $\mathcal{F} . \mathcal{F}$ should not query the Extract oracle with $I D^{*}$ as input and should not query the temporary private key $U_{I D^{*}}$ corresponding to $P_{I D^{*}}$.

Training Phase: The following oracle accesses are provided by $\mathcal{B}_{1}$ to $\mathcal{C}$.

IBS.Extract $\left(I D_{i}\right)$ : On input an identity $I D_{i}, \mathcal{B}_{1}$ returns the corresponding identity based private key $D_{i}$ if $I D \neq I D^{*}$. $\mathcal{B}_{1}$ maintains the list $L_{1}$ to store $\left\langle I D_{i}, D_{i}\right\rangle$.

IBS.Sig $\left(m, P_{i}, I D_{i}, Q_{i}\right)$ : With $\left(m, P_{i}, I D_{i}, Q_{i}\right)$ as input, $\mathcal{B}_{1}$ responds with an identity based signature $\sigma_{w a r}$ on the message $m$. $\mathcal{B}_{1}$ maintains $L_{2}$ to store the tuple $\left\langle m, P_{i}, I D_{i}, Q_{i}, \sigma_{w a r}\right\rangle$.

The following oracle access is provided by $\mathcal{B}_{2}$ to $\mathcal{C}$.

nonIBS.Sig $\left(m, I D_{i}, P_{i}\right)$ : On giving $\left(m, I D_{i}, P_{i}\right)$ as input, $\mathcal{B}_{2}$ responds with a non-identity based sign $\sigma_{s p}$ on message $m . \mathcal{B}_{2}$ maintains the list $L_{3}$ to store the tuple $\left\langle m, I D_{i}, P_{i}, \sigma_{s p}\right\rangle$.

$\mathcal{F}$ has access to all the following oracles during the EUF$G e n_{-} I B S P S$-CMA game, which are controlled by $\mathcal{C}$.

Extract $\left(I D_{i}\right)$ : In order to respond to this query by $\mathcal{F}, \mathcal{C}$ checks if $I D_{i} \neq I D^{*}$, if so then $\mathcal{C}$ queries $\mathcal{B}_{1}$ with $I D_{i}$ as input, i.e. queries IBS.Extract $\left(I D_{i}\right)$ to $\mathcal{B}_{1}$. $\mathcal{B}_{1}$ returns $D_{i}$ to $\mathcal{C}$. $\mathcal{C}$ stores the tuple $\left\langle I D_{i}, D_{i}\right\rangle$ into the list $F_{2}$ and returns $D_{i}$ to $\mathcal{F}$.

GenTempKey $\left(I D_{i}\right)$ : To respond to this query by $\mathcal{F}, \mathcal{C}$ fetches into the list $F_{1}$ for the tuple of the form $\left\langle i, P_{i}, U_{i}\right\rangle$ and returns both $\left(P_{i}, U_{i}\right)$ to $\mathcal{F}$.

WarrantSign $\left(m_{w}, P_{i}, I D_{i}, Q_{i}\right)$ : For this query by $\mathcal{F}, \mathcal{C}$ queries $\mathcal{B}_{2}$ for the identity based signature on $m_{w}$ with inputs $\left(m_{w}, P_{i}, I D_{i}, Q_{i}\right)$ as $\mathbf{I B S . S i g}\left(m_{w}, P_{i}, I D_{i}, Q_{i}\right)$. The response of the query, namely the signature $\sigma_{w a r}$ is returned to $\mathcal{C}$ and $\mathcal{C}$ sends it to $\mathcal{F}$.

$\operatorname{Proxy} \operatorname{Sign}\left(m, I D_{i}, P_{i}\right)$ : For this query by $\mathcal{F}, \mathcal{C}$ queries $\mathcal{B}_{2}$ as $\operatorname{nonIBS.Sig}\left(m, I D_{i}, P_{i}\right)$ for the non-identity based signature on $m$ with $\left(m, I D_{i}, P_{i}\right)$ as input. $\mathcal{B}_{2}$ returns $\sigma_{s p}$ to $\mathcal{C}$ which is send to $\mathcal{F}$ as response.

Forgery Phase: At the end of the training phase, $\mathcal{F}$ produces a forgery $\sigma^{*}=\left\langle m^{*}, m_{w}^{*}, \sigma_{w a r}^{*}, \sigma_{s p}^{*}\right\rangle$ and gives it to $\mathcal{C}$. $\mathcal{C}$ verifies the forgery and responds as follows:

Case 1: If $\mathcal{F}$ has not queried the WarrantSign oracle with $m_{w}^{*}, P_{I D^{*}}, I D^{*}, Q^{*}$ as input but has query the ProxySign 


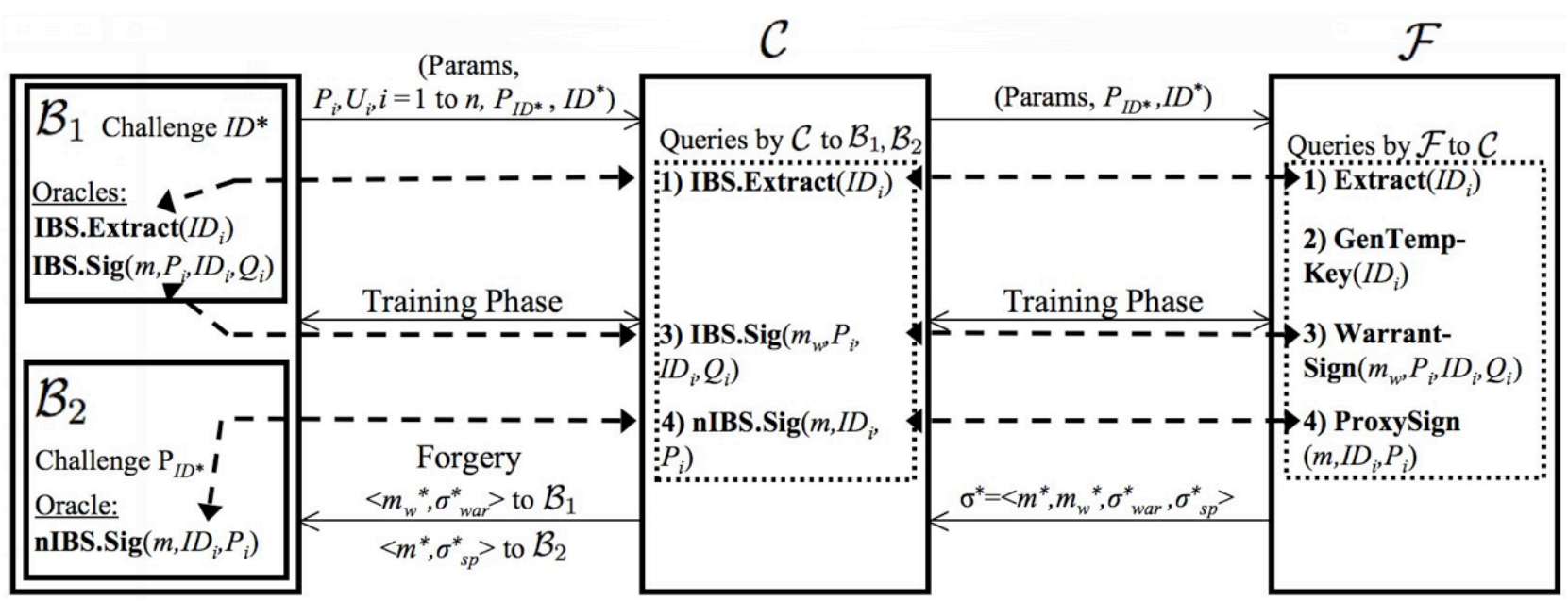

Figure 1. Relation among $\left(\mathcal{B}_{1}, \mathcal{B}_{2}\right), \mathcal{C}$ and $\mathcal{F}$

oracle with $m^{*}, I D^{*}, P_{I D^{*}}$ as input then $\mathcal{C}$ submits $\sigma_{\text {war }}^{*}$ to $\mathcal{B}_{1}$.

Case 2: If $\mathcal{F}$ has queried the WarrantSign oracle with $m_{w}^{*}, P_{I D^{*}}, I D^{*}, Q^{*}$ as input did not query the ProxySign oracle with $m^{*}, I D^{*}, P_{I D^{*}}$ as input then $\mathcal{C}$ submits $\sigma_{s p}^{*}$ to $\mathcal{B}_{2}$.

Analysis: Due to lack of space, we skip the formal probability analysis but the following is easy to see. Suppose $\epsilon$ is the advantage of $\mathcal{F}$ in the EUF-Gen_IBSPS-CMA game, which is denoted as $A d v_{\mathcal{F}}^{\text {wins }}=\epsilon$.

- If the forgery falls into Case $\mathbf{1}$, then $\mathcal{C}$ is capable of forging the underlying $I B S$ scheme with almost the same advantage $\epsilon$.

- If the forgery falls into Case 2, then $\mathcal{C}$ is capable of forging the underlying nonIBS scheme with almost the same advantage $\epsilon$.

\section{A CONCRETE IBSPS SCHEME}

In this section, we provide a concrete instantiation of Gen_IBSPS scheme and prove the security of the scheme in the proposed security model. We have used a variant of the $I B S$ signature scheme in [15] and the nonIBS scheme in [3], to construct our concrete IBSPS scheme.

\section{A. The Scheme}

The algorithms in the IBSPS scheme are described below:

\section{$\operatorname{Setup}\left(1^{\kappa}\right)$ :}

Let $\mathbb{G}_{1}$ be an additive group and $\mathbb{G}_{2}$ be a multiplicative group both of same prime order $q$. The PKG chooses a generator $P \in_{R} \mathbb{G}_{1}$, picks three cryptographic hash functions defined as $H_{1}:\{0,1\}^{*} \rightarrow \mathbb{G}_{1}$ and $H_{2}:\{0,1\}^{*} \times$ $\mathbb{G}_{1} \times \mathbb{G}_{1} \times \mathbb{G}_{1} \rightarrow \mathbb{G}_{1}$ and $H_{3}:\{0,1\}^{*} \times \mathbb{Z}_{q}^{*} \times \mathbb{G}_{1} \times \mathbb{G}_{1} \rightarrow \mathbb{G}_{1}$ and chooses a bilinear pairing $\hat{e}: \mathbb{G}_{1} \times \mathbb{G}_{1} \rightarrow \mathbb{G}_{2}$. The PKG computes $P_{\text {pub }}=s P$, where, $s \in_{R} \mathbb{Z}_{q}^{*}$ is the master private key.
$\operatorname{Extract}\left(I D_{A}\right)$ : Given a user's identity $I D_{A}$ as input the PKG computes $Q_{A}=H_{1}\left(I D_{A}\right) \in \mathbb{G}_{1}$ and $D_{A}=s H_{1}\left(I D_{A}\right)$ and sends $D_{A}$ to the user $A$.

GenTempKey $\left(\right.$ params, $\left.I D_{A}\right)$ : The signer generates his temporary private / public key pair by choosing $x_{A} \in \in_{R} \mathbb{Z}_{q}^{*}$ and computing $P_{A}=x_{A} P$. Now, the temporary private / public key pair of the user is $\left\langle P_{A}, U_{A}=x_{A}\right\rangle$.

WarrantSign $\left(m_{w}, P_{A}, I D_{A}, D_{A}\right)$ : The warrant signature on the warrant $m_{w}$ is generated as follows.

- Compute $R=r P$, where $r \in_{R} \mathbb{Z}_{q}^{*}$.

- $V_{w a r}=D_{A}+r H_{2}\left(m_{w}, R, Q_{A}, P_{A}\right)$.

- $\sigma_{w a r}=\left\langle V_{w a r}, R, P_{A}\right\rangle$ is the warrant signature.

Note: Warrant signature is independent of the message and hence need to be computed only once for the entire validity period of the warrant.

WarrantVerify $\left(m_{w}, I D_{A}, \sigma_{\text {war }}\right)$ : Given the identity $I D_{A}$ of the signer, a message warrant $m_{w}$ and a warrant signature $\sigma_{\text {war }}$ on $m_{w}$, this algorithm returns True if the following check holds; otherwise returns False.

$$
\hat{e}\left(V_{w a r}, P\right) \stackrel{?}{=} \hat{e}\left(R, H_{2}\left(m_{w}, R, P_{A}, Q_{A}\right)\right) \hat{e}\left(P_{p u b}, Q_{A}\right)
$$

$\operatorname{ProxySign}\left(m, I D_{A}, P_{A}, U_{A}\right)$ : Given a pair of temporary keys $\left\langle P_{A}, U_{A}\right\rangle$ the self proxy sign on a message $m$ with warrant $m_{w}$ by the user with identity $I D_{A}$, the proxy signature can be generated as

- $V_{s p}=x_{A} H_{3}\left(m, P_{A}, \alpha, I D_{A}\right)$ where $\alpha \in \in_{R} \mathbb{Z}_{q}^{*}$ (Note that $x_{A}=U_{A}$ ).

- $\sigma_{s p}$ is $\left\langle V_{s p}, \alpha\right\rangle$

$\operatorname{Proxy} V e r i f y\left(m, I D_{A}, P_{A}, \sigma_{s p}\right)$ : Given the Self Proxy signature $\sigma_{s p}$ and the temporary public key $P_{A}$ check whether:

$$
\hat{e}\left(V_{s p}, P\right) \stackrel{?}{=} \hat{e}\left(P_{A}, H_{3}\left(m, P_{A}, \alpha, I D_{A}\right)\right)
$$

If the check holds, return True; otherwise return False. 
$\operatorname{SPSSign}\left(m, m_{w}, I D_{A}, P_{A}, U_{A}, Q_{A}, D_{A}\right):$ In order to generate a self proxy signature $\sigma$, the user with identity $I D_{A}$, permanent key pair $\left(Q_{A}, D_{A}\right)$, temporary key pair $\left(P_{A}, U_{A}\right)$, a message warrant $m_{w}$ and a message $m$ to be signed, the user performs the following:

- $\sigma_{w a r} \leftarrow$ WarrantSign $\left(m_{w}, P_{A}, I D_{A}, D_{A}\right)$.

- $\sigma_{s p} \leftarrow \operatorname{ProxySign}\left(m, P_{A}, I D_{A}, U_{A}\right)$

Output the self proxy signature $\sigma=\left\langle\sigma_{w a r}, \sigma_{s p}\right\rangle$.

Note: If the warrant sign $\sigma_{w a r}$ on the tuple $\left(m_{w}, P_{A}, I D_{A}, D_{A}\right)$ was already generated this need not be recomputed for every message, the signer need to call only the algorithm ProxySign $\left(m, P_{A}, I D_{A}, U_{A}\right)$ to generate $\sigma_{s p}$.

$\operatorname{SPSVerify}\left(m, m_{w}, Q_{A}, P_{A}, \sigma\right)$ : If the output of the algorithm WarrantVerify $\left(m_{w}, I D_{A}, \sigma_{w a r}\right)=$ True and $\operatorname{ProxyVerify}\left(m, I D_{A}, P_{A}, \sigma_{s p}\right)=$ True then output True else output False.

\section{B. Security Proof for IBSPS}

Theorem 2: If there exists a forger $\mathcal{F}$ who is capable of breaking the EUF-IBSPS-CMA security of the IBSPS scheme with non-negligible advantage, then we can efficiently construct an algorithm $\mathcal{C}$, which can solve $C D H P$ with almost the same advantage of $\mathcal{F}$.

Proof: The challenger $\mathcal{C}$ is given a random instance of CDHP, say $(P, a P, b P), \mathcal{C}$ 's aim is to compute $a b P$. Let us assume that there is a forger $\mathcal{F}$ who is capable of breaking the EUF-IBSPS-CMA security of our identity based self proxy signature scheme IBSPS. $\mathcal{C}$ simulates the system with the following oracles $\mathrm{H}_{1}, \mathrm{H}_{2}, \mathrm{H}_{3}$, Extract, GenTempKey, WarrantSign, ProxySign and SPSSign. The forger $\mathcal{F}$ can query these oracles which are controlled by the challenger $\mathcal{C}$. Each oracle maintains a list to maintain the consistency of the replies to the queries.

Setup: $\mathcal{C}$ sets up the system parameters in the following way.

- $\mathcal{C}$ chooses the groups $\mathbb{G}_{1}$ and $\mathbb{G}_{2}$ and the generator $P \in \mathbb{G}_{1}$ as given in the CDHP instance.

- Sets the master public key $P_{p u b}=b P$, which is a part of $C D H P$ instance. It is to be noted that $\mathcal{C}$ does not know $b$.

- Models all the hash functions as random oracles.

- Selects a bilinear map $\hat{e}: \mathbb{G}_{1} \times \mathbb{G}_{1} \rightarrow \mathbb{G}_{2}$.

- Delivers $\left(\hat{e}, \mathbb{G}_{1}, \mathbb{G}_{2}, P, P_{\text {pub }}\right)$ to $\mathcal{F}$ as params.

Training Phase: $\mathcal{F}$ performs polynomially bounded number of queries, in an adaptive manner (i.e., each query may depend on the responses to the previous queries) during this phase.

$H_{1}$ Oracle: Let $q_{H 1}$ be the number of queries asked by $\mathcal{F}$. $\mathcal{C}$ selects a random index $\gamma$, where $1 \leq \gamma \leq q_{H 1}$. $\mathcal{C}$ doesn't reveal $\gamma$ to $\mathcal{F}$. When $\mathcal{F}$ generates the $\gamma^{\text {th }}$ query to this oracle, $\mathcal{C}$ decides to fix the corresponding identity $\left(I D_{\gamma}\right)$ as the target identity for the challenge phase. $\mathcal{C}$ maintains a list $L_{1}$ to consistently reply the $H_{1}$ oracle queries. $\mathcal{C}$ replies as follows.

- Searches in list $L_{1}$ and checks whether a matching tuple corresponding to $I D_{i}$ exists. If it exists, $\mathcal{C}$ returns the value $x_{i} P$.

- Otherwise, $\mathcal{C}$ performs the following:

- If $I D_{i}=I D_{\gamma}$ then, sets $H_{1}\left(I D_{i}\right)=a P$ and adds tuple $\left\langle I D_{\gamma}, a P, \perp, \perp\right\rangle$ to $L_{1}$.

- If $I D_{i} \neq I D_{\gamma}$ then, chooses $x_{i} \in_{R} \mathbb{Z}_{q}^{*}$, sets $H_{1}\left(I D_{i}\right)=x_{i} P$, computes $D_{i}=x_{i} P_{p u b}$ and adds the tuple $\left\langle I D_{i}, x_{i} P, x_{i}, D_{i}\right\rangle$ to the list $L_{1}$.

$H_{2}$ Oracle: $\left(m_{w}, R, P_{i}, Q_{i}\right)$. Let $L_{2}$ be the list associated with this oracle. If the oracle was not queried previously with $\left(m_{w}, R, P_{i}, Q_{i}\right)$ as input, $\mathcal{C}$ chooses $r \in_{R} \mathbb{Z}_{q}^{*}$, computes $\mathrm{H}_{2}^{i}=r P$, adds the tuple $\left\langle m_{w}, r, R, P_{i}, Q_{i}, \mathrm{H}_{2}^{i}\right\rangle$ to the list $L_{2}$ and returns $\mathrm{H}_{2}^{i}$ to $\mathcal{F}$. If the tuple $\left\langle m_{w}, r, R, P_{i}, Q_{i}\right\rangle$ already exists in $L_{2}$ then returns the corresponding $\mathrm{H}_{2}^{i}$ to $\mathcal{F}$.

$H_{3}$ Oracle: $\left(m, I D_{i}, \alpha, P_{i}\right)$. Let $L_{3}$ be the list associated with this oracle. If $\left(m, I D_{i}, \alpha, P_{i}\right)$ was queried previously, $\mathcal{C}$ returns $\mathrm{H}_{3}^{i}$ retrieved from the tuple $\left\langle m, I D_{i}, \alpha, \perp, \mathrm{H}_{3}^{i}\right\rangle$ which is already stored in the list $L_{3}$. If the tuple does not exist, $\mathcal{C}$ performs the following:

- If $I D_{i} \neq I D_{\gamma}$ then, chooses $\mathrm{H}_{3}^{i} \in_{R} \mathbb{G}_{1}$, adds the tuple $\left\langle m, I D_{i}, \alpha, P_{i}, \perp, \mathrm{H}_{3}^{i}\right\rangle$ to the list $L_{3}$ and returns $\mathrm{H}_{3}^{i}$ to $\mathcal{F}$.

- If $I D_{i}=I D_{\gamma}$ then, chooses $z \in_{R} \mathbb{Z}_{q}^{*}$, computes $\mathrm{H}_{3}^{i}=z b P$ adds the tuple $\left\langle m, I D_{i}, \alpha, P_{i}, z, \mathrm{H}_{3}^{i}\right\rangle$ to the list $L_{3}$ and returns $\mathrm{H}_{3}^{i}$ to $\mathcal{F}$.

Extract Oracle $\left(I D_{i}\right)$ : For any given identity $I D_{i} \neq I D_{\gamma}, \mathcal{C}$ searches for the private key $D_{i}$ in list $L_{1}$, corresponding to $I D_{i}$ and returns it to $\mathcal{F}$. If $I D_{i}=I D_{\gamma}, \mathcal{F}$ aborts.

WarrantSign Oracle $\left(m_{w}, I D_{i}, P_{i}\right): \mathcal{F}$ queries the warrant signature on a warrant $m_{w}$ for a signer with identity $I D_{i}$.

- If $I D_{i} \neq I D_{\gamma}, \mathcal{C}$ responds as per the WarrantSign algorithm by creating new temporary keys $U_{i}=k_{i}$, $P_{i}=k_{i} P$, where $k_{i} \in \in_{R} \mathbb{Z}_{q}^{*} \cdot \mathcal{C}$ adds the tuple $\left\langle I D_{i}, P_{i}, k_{i}\right\rangle$ to the list $L_{4}$.

- If $I D_{i}=I D_{\gamma}, \mathcal{C}$ responds as follows.

- Chooses $k, y \in \in_{R} \mathbb{Z}_{q}^{*}$.

- Computes $V_{w a r}=y k P_{p u b}$ and $P_{i}=k a P$.

- Computes $R=k P_{p u b}$ and $\mathrm{H}_{2}^{i}=-k^{-1} Q_{i}+y P$. In this case $\mathcal{C}$ does not query the $H_{2}$ oracle, instead it sets the value for the hash computation and stores it in the list.

- Returns the signature $\sigma_{w a r}=\left(m_{w}, V_{w a r}, P_{i}\right)$ to $\mathcal{F}$.

The correctness of $\sigma_{w a r}$ follows from the validity check in the WarrantVerify algorithm : $\hat{e}\left(V_{\text {war }}, P\right) \stackrel{?}{=} \hat{e}\left(R, H_{2}\left(w, R, P_{A}, Q_{i}\right)\right) \hat{e}\left(P_{p u b}, Q_{i}\right)$. Here, the L.H.S $\hat{e}\left(V_{w a r}, P\right)=\hat{e}(y k b P, P)$ 


$$
\begin{aligned}
\text { R.H.S } & =\hat{e}(H, R) \hat{e}\left(Q_{i}, P_{p u b}\right) \\
& =\hat{e}\left(-k^{-1} Q_{i}+y P, k b P\right) \hat{e}(a P, b P) \\
& =\hat{e}\left(k\left(-k^{-1} Q_{i}+y P\right), b P\right) \hat{e}(a P, b P) \\
& =\hat{e}(-a P+y k P, b P) \hat{e}(a P, b P) \\
& =\hat{e}(y k P, b P)=\hat{e}(y k b P, P)=\text { L.H.S }
\end{aligned}
$$

Thus, it is clear that $\sigma_{\text {war }}$ generated in this way is a valid signature by user with identity $I D_{i}$ on warrant $m_{w}$.

GenTempKey Oracle $\left(I D_{i}\right): \mathcal{F}$ produces an identity $I D_{i}$ to $\mathcal{C}$ and queries the corresponding the temporary private, public keys $U_{i}$ and $P_{i}$. $\mathcal{C}$ responds to $\mathcal{F}$ as follows:

- If $I D_{i} \neq I D_{\gamma}$ then, $\mathcal{C}$ chooses $k_{i} \in_{R} \mathbb{Z}_{q}^{*}$, set $P_{i}=k_{i} P$, adds the tuple $\left\langle I D_{i}, P_{i}, k_{i}\right\rangle$ to list $L_{4}$ and returns $P_{i}$.

- If $I D_{i}=I D_{\gamma}$ then, $\mathcal{C}$ chooses $k_{i} \in R \mathbb{Z}_{q}^{*}$, set $P_{i}=$ $k_{i} a P$, adds $\left\langle I D_{i}, P_{i}, k_{i}\right\rangle$ to list $L_{4}$ and returns $P_{i}$.

ProxySign Oracle $\left(m, I D_{i}, P_{i}\right): \mathcal{F}$ queries this oracle with $\left(m, I D_{i}, P_{i}\right)$ where $I D_{i}$ is signer's identity, $P_{i}$ is the signer's temporary public key and $m$ is the message to be signed, $\mathcal{C}$ searches $L_{4}$ and retrieves the tuple $\left\langle I D_{i}, U_{i}, P_{i}\right\rangle$ and responds as follows:

- If the signer's identity $I D_{i} \neq I D_{\gamma}$, the challenger $\mathcal{C}$ proceeds as per the ProxySign algorithm.

- If $I D_{i}=I D_{\gamma}, \mathcal{C}$ performs the following:

- Chooses $z, \alpha \in_{R} \mathbb{Z}_{q}^{*}$.

- Computes $V_{s p}=z P_{i}$

- Computes $\mathrm{H}_{3}^{i}=z P$ and stores the tuple $\left\langle m, I D_{i}, \alpha, P_{i}, \perp, \mathrm{H}_{3}^{i}\right\rangle$ in list $L_{3}$. (Note that $\mathcal{C}$ did not query $H_{3}$ oracle, instead it sets the value for the hash computation)

- Returns $\sigma_{s p}=\left(m, V_{s p},\right)$

Forgery Phase: At the end of the training phase, $\mathcal{F}$ produces a forgery $\sigma^{*}=\left\langle m^{*}, m_{w}^{*}, \sigma_{w a r}^{*}, \sigma_{s p}^{*}\right\rangle$ on identity $I D^{*}$ and gives $\sigma^{*}$ and $I D^{*}$ to $\mathcal{C}$. Here $m_{w}^{*}$ is the message warrant, $m^{*}$ is the message, $I D^{*}$ is the identity of the signer, $\sigma_{\text {war }}^{*}=\left\langle V_{\text {war }}^{*}, R^{*}, P_{I D^{*}}\right\rangle$ is the warrant signature and $\sigma_{s p}^{*}=\left\langle V_{s p}^{*}, \alpha^{*}\right\rangle$ is the proxy signature. $\mathcal{C}$ verifies the forgery and obtains the solution for the CDHP instance in either one of the following cases:

Case 1: Assume that $\mathcal{F}$ has not queried the WarrantSign oracle with $\left(m_{w}^{*}, P_{I D^{*}}, I D^{*}, Q^{*}\right)$ as input but queried the ProxySign oracle with $\left(m^{*}, I D^{*}, P_{I D^{*}}\right)$ as input. $\mathcal{C}$ makes use of $\sigma_{\text {war }}^{*}$ to solve the CDHP instance as follows:

- $\sigma_{w a r}^{*}=\left\langle V_{w a r}^{*}, R^{*}, P_{I D^{*}}\right\rangle$ is the warrant signature.

- $\mathcal{C}$ retrieves the tuple $\left\langle m, r, R, P_{i}, Q_{i}, \mathrm{H}_{2}^{i}\right\rangle$ from the list $L_{2}$ and checks whether $\hat{e}\left(V_{\text {war }}^{*}, P\right) \stackrel{?}{=} \hat{e}(a P, b P) \hat{e}\left(\mathrm{H}_{2}^{i}, P\right)$.

- If the above check holds then $\mathcal{C}$ computes $V_{\text {war }}^{*}-r P=$ $a b P$.

The above computation is correct because $\mathcal{C}$ has set $P_{\text {pub }}=$ $b P$ and the public key of $I D_{\gamma}$ as $a P$. Moreover, $\mathcal{C}$ has set $\mathrm{H}_{2}^{i}=r P$ corresponding to the message, which is retrievable from the list $L_{2}$. Thus, $V_{w a r}^{*}=a b P+r P$ and computing $V_{w a r}^{*}-r P$ reveals $a b P$.
Case 2: Assume that $\mathcal{F}$ has queried the WarrantSign oracle with $\left(m_{w}^{*}, P_{I D^{*}}, I D^{*}, Q^{*}\right)$ as input but did not query the ProxySign oracle with $\left(m^{*}, I D^{*}, P_{I D^{*}}\right)$ as input. $\mathcal{C}$ makes use of $\sigma_{s p}^{*}$ to solve the CDHP instance as follows:

- $\sigma_{s p}^{*}=\left\langle V_{s p}^{*}, \alpha^{*}\right\rangle$ is the proxy signature.

- $\mathcal{C}$ knows that $V_{s p}^{*}=k_{i} z a b P$. This is because the GenTempKey oracle has set $P_{i}=k_{i} a P$ and the $H_{3}$ oracle has set $\mathrm{H}_{3}^{i}=z b P$ for the corresponding $\alpha^{*}$, when $I D_{i}=I D_{\gamma}$.

- $\mathcal{C}$ now retrieves the tuple $\left\langle I D_{i}, P_{i}, k_{i}\right\rangle$ from the list $L_{4}$ and the tuple $\left\langle m, I D_{i}, \alpha, P_{i}, z, \mathrm{H}_{3}^{i}\right\rangle$ from the list $L_{3}$.

- Checks whether $\alpha=\alpha^{*}$ and $\hat{e}\left(V_{s p}^{*}, P\right) \stackrel{?}{=} \hat{e}\left(P_{i}, \mathrm{H}_{3}^{i}\right)$.

- If the above check holds then, $\mathcal{C}$ computes $z^{-1} k_{i}^{-1} V_{s p}^{*}=a b P$.

Analysis: Let $\mathcal{E}_{1}$ be the event in which $\mathcal{C}$ aborts when $\mathcal{F}$ queries the private key corresponding to $I D^{*}$ and $\mathcal{E}_{2}$ be the event in which $I D_{\gamma}$ is not chosen as the target identity by $\mathcal{F}$ for generating the forgery. Suppose $\mathcal{F}$ has made $q_{H_{1}}$ number of $H_{1}$ Oracle queries and $q_{E}$ number of Extract Oracle queries, then:

$$
\operatorname{Pr}\left[\mathcal{E}_{1}\right]=\frac{q_{E}}{q_{H_{1}}} \text { and } \operatorname{Pr}\left[\mathcal{E}_{2}\right]=\frac{1}{q_{H_{1}}-q_{E}} .
$$

Therefore, $\quad \operatorname{Pr}\left[\mathcal{F}_{E U F-I B S P S-C M A}^{\text {wins }}\right]=\left[\neg \mathcal{E}_{1} \wedge \mathcal{E}_{2}\right]=$ $\left[1-\frac{q_{E}}{q_{H_{1}}}\right] \cdot\left[\frac{1}{q_{H_{1}}-q_{E}}\right]=\frac{1}{q_{H_{1}}}$.

Thus the challenger $\mathcal{C}$ solves the $C D H P$ instance with almost the same probability as the forger $\mathcal{F}$ wins the EUFIBSPS-CMA game.

\section{CONCLUSION}

We have introduced the notion of identity based self proxy signature scheme, wherein a signer creates temporary private key / public key pair which is controlled by a corresponding message warrant. The message warrant and the temporary public key are signed with the permanent identity based private key of the signer and the signature on the message is signed with the temporary private key. The temporary private key is revoked in appropriate time intervals. We have given a generic construction for identity based SPS, proposed the formal security model, given a concrete instantiation and proved it in the random oracle model. Several specific schemes can be constructed by choosing a specific identity based scheme for warrant signing and a non-identity based signature scheme for message signing.

For example, in section VI-A, we have used a variant of the $I B S$ signature scheme in [15] for warrant signing and the nonIBS scheme in [3] for message signing. Other possible combinations are, we may use CC [4], FH [6], SOK [15] for warrant signing and Schnorr, EC-DSA, BLS [3] signatures for message signing. The table in Fig. 4. summarizes the complexity figures for each of this combination. We observe that, if self proxy signatures are generated in a 


\begin{tabular}{|l|c|c|c|c|c|c|c|c|}
\hline \multirow{3}{*}{ Scheme } & \multicolumn{4}{|c|}{ One Signature } & \multicolumn{4}{c|}{$\begin{array}{c}\text { Signatures } \\
\text { (During a Session) }\end{array}$} \\
\cline { 2 - 10 } & \multicolumn{2}{|c|}{ Sign } & \multicolumn{2}{c|}{ Verify } & \multicolumn{2}{c|}{ Sign } & \multicolumn{2}{c|}{ Verify } \\
\cline { 2 - 9 } & PM & BP & PM & BP & PM & BP & PM & BP \\
\hline CC [4] & 2 & - & 1 & 2 & $2 n$ & - & $n$ & $2 n$ \\
\hline FH [6] & 3 & 1 & 1 & 2 & $3 n$ & $n$ & $n$ & $2 n$ \\
\hline SOK [14] & 2 & - & - & 3 & $2 n$ & - & - & $3 n$ \\
\hline
\end{tabular}

Figure 2. Complexity Figure for IBS Schemes per Signature and per Session

\begin{tabular}{|l|c|c|c|c|}
\hline \multirow{2}{*}{ Scheme } & \multicolumn{3}{c|}{ One Signature } \\
\cline { 2 - 5 } & \multicolumn{2}{|c|}{ Sign } & \multicolumn{2}{c|}{ Verify } \\
\cline { 2 - 5 } & PM & BP & PM & BP \\
\hline Schnorr & 1 & - & 2 & - \\
\hline EC-DSA & 1 & - & 2 & - \\
\hline BLS [3] & 1 & - & - & 2 \\
\hline
\end{tabular}

Figure 3. Complexity Figure for nonIBS Schemes per Signature

\begin{tabular}{|c|c|c|c|c|c|c|c|c|c|}
\hline \multicolumn{2}{|c|}{ Scheme } & \multicolumn{4}{|c|}{ One Signature } & \multicolumn{4}{|c|}{$\begin{array}{c}n \text { Signatures (During a } \\
\text { Session) }\end{array}$} \\
\hline \multirow{2}{*}{$\begin{array}{r}\text { Warrant } \\
\text { Sign (IBS) }\end{array}$} & \multirow{2}{*}{$\begin{array}{l}\text { Proxy Sign } \\
\text { (nIBS) }\end{array}$} & \multicolumn{2}{|c|}{ Sign } & \multicolumn{2}{|c|}{ Verify } & \multicolumn{2}{|c|}{ Sign } & \multicolumn{2}{|c|}{ Verify } \\
\hline & & PM & BP & PM & BP & PM & BP & PM & BP \\
\hline \multirow{3}{*}{ CC [4] } & Schnorr & $2+1$ & - & $1+1$ & $2+0$ & $2+n$ & - & $1+n$ & $2+0$ \\
\hline & EC-DSA & $2+1$ & - & $1+1$ & $2+0$ & $2+n$ & & $1+n$ & $2+0$ \\
\hline & BLS [3] & $2+1$ & - & $1+0$ & $2+2$ & $2+n$ & & $1+0$ & $2+2 n$ \\
\hline \multirow{3}{*}{ FH [6] } & Schnorr & $3+1$ & 1 & $1+1$ & $2+0$ & $3+n$ & 1 & $1+n$ & $2+0$ \\
\hline & EC-DSA & $3+1$ & 1 & $1+1$ & $2+0$ & $3+n$ & 1 & $1+n$ & $2+0$ \\
\hline & BLS [3] & $3+1$ & 1 & $1+0$ & $2+2$ & $3+n$ & 1 & $1+0$ & $2+2 n$ \\
\hline \multirow{3}{*}{ SOK [14] } & Schnorr & $2+1$ & - & - & $3+0$ & $2+n$ & - & - & $3+0$ \\
\hline & EC-DSA & $2+1$ & - & - & $3+0$ & $2+n$ & - & - & $3+0$ \\
\hline & BLS [3] & $2+1$ & - & - & $3+2$ & $2+n$ & - & - & $3+2 n$ \\
\hline
\end{tabular}

Figure 4. Complexity Figure of IBSPS per Signature and per Session Legend - [BP - Bilinear Pairing, PM - Scalar Point Multiplication] 
session the total operation count turns out to be $n+c$ for some constant $c$ for our schemes. For example, if signatures on warrant is generated using $\mathrm{CC}$ [4] and the proxy signature is generated by Schnorr scheme, then the total number of scalar point multiplication is $2+n$ for signing and $1+n$ for verifying (See row 1 of Fig. 4.). However, direct application of CC [4] scheme results in $2 n$ scalar point multiplication during signing and $n$ during verification. Thus our method has significantly reduced the computational complexity from $2 n$ to $n+2$ during signing. The complexity figure for other combination of schemes in our generic scheme is given in Fig. 4.

\section{REFERENCES}

[1] Paulo S. L. M. Barreto, Benoît Libert, Noel McCullagh, and Jean-Jacques Quisquater. Efficient and provably-secure identity-based signatures and signcryption from bilinear maps. In Advances in cryptology ASIACRYPT05, volume 3788 of Lecture Notes in Computer Science, pages 515-532. Springer, 2005.

[2] Alexandra Boldyreva, Adriana Palacio, and Bogdan Warinschi. Secure proxy signature schemes for delegation of signing rights. Cryptology ePrint Archive, Report 2003/096, 2003. eprint.iacr.org.

[3] Dan Boneh, Ben Lynn, and Hovav Shacham. Short signatures from the weil pairing. In Advances in Cryptology - ASIACRYPT 2001, volume 2248 of Lecture Notes in Computer Science, pages 514-532. Springer, 2001.

[4] Jae Choon Cha and Jung Hee Cheon. An identity-based signature from gap diffie-hellman groups. In Public Key Cryptography - PKC 2003, volume 2567 of Lecture Notes in Computer Science, pages 18-30. Springer, 2003.

[5] Oded Goldreich, Birgit Pfitzmann, and Ronald L. Rivest. Selfdelegation with controlled propagation - or - what if you lose your laptop. In Advances in Cryptology - CRYPTO 1998, volume 1462 of Lecture Notes in Computer Science, pages 153-168. Springer, 1998.

[6] Florian Hess. Efficient identity based signature schemes based on pairings. In Selected Areas in Cryptography, SAC - 2002, volume 2595 of Lecture Notes in Computer Science, pages 310-324. Springer, 2003.

[7] SHIN-JIA Hwang and CHIU-CHIN Chen. New multiproxy multi-signature schemes. Applied mathematics and computation, Elsevier, New York, vol. 147(no. 1):57-67, 2004.

[8] SHIN-JIA Hwang and C. Shi. A simple multi-proxy signature scheme. In Proceedings of the $10^{t} \mathrm{~h}$ National Conference on Information Security, pages 134-138, 2000.

[9] Seungjoo Kim, Sangjoon Park, and Dongho Won. Proxy signatures, revisited. In Information and Communication Security (ICICS - 1997), volume 1334 of Lecture Notes in Computer Science, pages 223-232. Springer, 1997.

[10] Young-Seol Kim and Jik Hyun Chang. Self proxy signature scheme. IJCSNS International Journal of Computer Science and Network Security, vol. 7(no. 2):335-338, 2007.
[11] Byoungcheon Lee, Heesun Kim, and Kwangjo Kim. Strong proxy signature and its applications. In The 2001 Symposium on Cryptography and Information Security - SCIS 2001, 2001.

[12] Younho Lee, Heeyoul Kim, Yongsu Park, and Hyunsoo Yoon. A new proxy signature scheme providing self-delegation. In Information Security and Cryptology - ICISC 2006, volume 4296 of Lecture Notes in Computer Science, pages 328-342. Springer, 2006.

[13] Tal Malkin, Satoshi Obana, and Moti Yung. The hierarchy of key evolving signatures and a characterization of proxy signatures. In Advances in Cryptology - EUROCRYPT 2004, volume 3027 of Lecture Notes in Computer Science, pages 306-322. Springer, 2004.

[14] Masahiro Mambo, Keisuke Usuda, and Eiji Okamoto. Proxy signatures for delegating signing operation. In ACM Conference on Computer and Communications Security ACMCCS96, pages 48-57, 1996.

[15] R. Sakai, K. Ohgishi, and M. Kasahara. Cryptosysytems based on pairing. In In Symposium on Cryptography and Information Security - SCIS 2000, 2000.

[16] Adi Shamir. Identity-based cryptosystems and signature schemes. In CRYPTO - 1984, pages 47-53. Springer, 1984.

[17] Lijang Yi, Guoqiang Bai, and Guozhen Xiao. Proxy multisignature scheme: A new type of proxy signature scheme. Electronic letters, vol. 36(Issue. 6):527-528, 2000. 